\title{
BSG erschwert Nachbesetzung von Arztstellen
}

Das Urteil des Bundessozialgerichts (BSG) vom 04.05.2016 (Az.: B 6 KA 21/15) schlägt hohe Wellen. Denn das BSG schiebt der bisherigen Spruchpraxis der Zulassungsgremien bei der Nachbesetzung von Arztstellen, die durch Verzicht eines niedergelassenen Vertragsarztes zu Gunsten einer Anstellung entstanden sind, einen Riegel vor. Im Grundsatz soll eine Nachbesetzung nur noch möglich sein, wenn der (ursprünglich verzichtende) Arzt beabsichtigt, 3 Jahre auf der Arztstelle angestellt zu sein.

\section{Das Urteil}

Ausgangspunkt für das Urteil war zwar die Nachbesetzung einer Arztstelle. Gegenstand des Rechtsstreits war jedoch eigentlich die Frage, ob die Stelle eines ehemaligen Vertragsarztes, der seine Tätigkeit als Angestellter im MVZ von Anfang an nur im Umfang einer Teilzeittätigkeit aufgenommen hat, gleichwohl mit einer vollen Stelle nachbesetzt werden könnte. Das BSG lehnte dies mit Hinweis auf eine Umgehung der für die Nachfolgezulassung geltenden Voraussetzungen ab.

Das BSG nutzte diesen Fall, um sein Verständnis der grundsätzlichen Rahmenbedingungen bei der Übertragung von Zulassungen im gesperrten Bereich darzulegen - mit weitreichenden Folgen in der Praxis.

Sehr dezidiert und systematisch legt das BSG in dem Urteil die verschiedenen Gestaltungsmöglichkeiten - die das SGB
V für Vertragsärzte, die ihre Zulassung auf- oder im Rahmen eines Praxisverkaufs weitergeben wollen, vorsieht - dar.

\section{Variante 1:}

\section{Verzicht und Ende der Zulassung}

Zunächst kann der Arzt es bei der Erklärung des Verzichts auf die Zulassung bewenden lassen. Dies hat zur Folge, dass die Zulassung ersatzlos entfällt und sich der Grad der (Über-)Versorgung entsprechend reduziert.

\section{Variante 2: \\ Verzicht und Ausschreibung}

Der Vertragsarzt kann aber auch den Verzicht auf die Zulassung mit einem Antrag auf Durchführung eines Verfahrens zur Nachbesetzung des Praxissitzes verbinden. Bewerben sich nach positiver Entscheidung über die Durchführung eines Nachbesetzungsverfahrens mehrere Ärzte um die Praxisnachfolge, sieht das
SGB V eine Auswahlentscheidung des Zulassungsausschusses vor, zitiert das BSG die rechtlichen Grundlagen. Um die Praxisnachfolge kann sich auch ein MVZ bewerben. Das SGB V sieht ausdrücklich die Möglichkeit einer Weiterführung der Praxis in der Form vor, dass ein MVZ den Vertragsarztsitz übernimmt und die vertragsärztliche Tätigkeit durch einen angestellten Arzt in der Einrichtung weiterführt. (Eine entsprechende Regelung gibt es auch für Vertragsärzte.) Die Auswahl des angestellten Arztes obliegt in diesem Fall zwar dem MVZ bzw. dem Vertragsarzt. Die Entscheidung, ob das MVZ bzw. der anstellende Vertragsarzt die Nachfolge antritt oder ob ein anderer Bewerber um die Nachfolge ausgewählt wird, trifft jedoch der Zulassungsausschuss.

\section{Variante 3: Verzicht zugunsten einer Anstellung}

Eine dritte Möglichkeit besteht darin, dass der Vertragsarzt, der auf seine Zulassung verzichtet, selbst als Angestellter entweder bei einem Vertragsarzt oder in einem MVZ tätig wird. Für diesen Fall ist weder eine Entscheidung des Zulassungsausschusses, ob eine Nachbesetzung erfolgen kann noch eine Auswahlentscheidung zwischen mehreren Bewerbern um die Praxisnachfolge vorgesehen. Vielmehr ist dem MVZ die Genehmigung zur Anstellung des Arztes zu erteilen, der auf seine Zulassung verzichtet hat.

Diese - gesetzlich vorgesehene - Möglichkeit wurde seit ihrer Einführung von vielen MVZ genutzt, da hierdurch Vertragsarztsitze sicher in ein MVZ integriert werden konnten, ohne dass ein Kampf um den Vertragsarztsitz (bei mehreren Bewerbern) bei den Zulassungsgremien befürchtet werden musste. Je nach Bezirk der Zulassungsgremien entwickelte sich eine eigene Spruchpraxis dahingehend, dass die so integrierten Arztstellen z.B. nach einem oder 2 Quartalen, in denen der verzichtende Arzt die Arztstelle auch tatsächlich zu „bedienen“ hatte, durch Ј konnte. Dieses Vorgehen wurde daher gerade für Vertragsarztsitze älterer Ärzte bevorzugt, die ihre Praxis verkaufen und die Risiken der Ausschreibungen umgehen wollten. Auf der anderen Seite war es auch ein beliebtes Vorgehen für Träger 
von MVZ, um Vertragsarztsitze unkompliziert in die MVZ zu ziehen und kurzfristig mit „eigenen“ Leuten nachzubesetzen.

\section{Voraussetzung: \\ Arzt bleibt im System}

$\mathrm{Zu}$ dieser letzten Variante äußerte sich nun das BSG erstmals mit inhaltlichen Voraussetzungen, dem die bisherige Praxis offensichtlich ein Dorn im Auge war. Entscheidende Voraussetzung für die Privilegierung dieser Variante (nämlich keine Ausschreibung und kein Auswahlverfahren) ist nach Auffassung des BSG gemäß dem SGB V, dass der Arzt auf seine Zulassung verzichtet hat, „um in einem medizinischen Versorgungszentrum tätig zu werden“ (so der Gesetzeswortlaut). Der entscheidende Unterschied zur zweiten Variante und der Grund dafür, dass die Anstellungsgenehmigung beim Vorliegen der gesetzlichen Voraussetzungen zu erteilen sei, ohne dass dem Zulassungsausschuss ein Entscheidungsspielraum verbleibe, liege darin, dass der Vertragsarzt bei dieser Variante des Zulassungsverzichts seine Tätigkeit innerhalb des Systems der vertragsärztlichen Versorgung und nur mit einem anderen Status - dem des Angestellten fortführe. Diese dritte Gestaltung des Verzichts auf die Zulassung sei deshalb von vornherein auf eine Weiterführung der Versorgung und nicht auf ein Ausscheiden aus dem System angelegt, so der Senat.

Daraus folge, dass MVZ und Vertragsärzte die Privilegien der dritten Variante grundsätzlich nur in Anspruch nehmen könnten, wenn und soweit der Arzt auf seine Zulassung gerade mit dem Ziel verzichte, selbst in dem MVZ oder beim Vertragsarzt als angestellter Arzt tätig zu werden; es werde also Personenidentität zwischen dem auf die Zulassung verzichtenden Arzt und dem Arzt vorausgesetzt, der die Anstellung in dem MVZ aufnimmt.

\section{BSG: Geringe Dauer der}

Anstellung reicht nicht aus

Aus der Tatsache, dass eine Anstellungsgenehmigung nach $\S 103$ Abs. 4a Satz 1 SGB V nur erteilt werden darf, wenn der anzustellende Arzt gerade mit dem Ziel auf seine Zulassung verzichtet hat, in dem MVZ tätig zu werden, aus dem Ausnahmecharakter der Regelung sowie den im Urteil dargestellten systematischen Erwägungen folgert das BSG, dass nicht jede beliebig geringe Dauer einer angestrebten Angestelltentätigkeit den Anspruch auf die Erteilung einer Genehmigung nach dieser Vorschrift begründen könne. Nur wie legt man die erforderliche Dauer fest? Als innerer Vorgang sei die Absicht des auf die Zulassung verzichtenden Arztes, in dem MVZ tätig zu werden, einer objektiven Überprüfung nicht ohne weiteres zugänglich. Zweifellos werde sich der Wille des Arztes, in einem MVZ tätig zu werden, aber jedenfalls nicht in einer „logischen Sekunde“ erschöpfen dürfen; vielmehr müsse die Tätigkeit als Angestellter tatsächlich ausgeübt werden, weil unter diesen Umständen feststehe, dass nicht der Wille des auf die Zulassung verzichtenden Arztes im Vordergrund stehe, im MVZ tätig zu werden, sondern der Wille des MVZ, die Stelle nach eigener Wahl nachbesetzen zu können.

\section{Nicht ausreichend: logische} Sekunde oder ein bis 2 Quartale

Konkrete Hinweise auf den zeitlichen Horizont, auf den sich der Wille zur Ausübung der Tätigkeit als Angestellter im MVZ zu beziehen habe, damit daraus auf einen entsprechenden Willen geschlossen werden kann, seien dem Wortlaut der Vorschrift allerdings nicht zu entnehmen, so die Richter. Eine Dauer der Angestelltentätigkeit von ein oder 2 Quartalen reiche jedoch nicht aus, um zu dokumentieren, dass auf die Zulassung gerade mit dem Ziel verzichtet wurde, in einem MVZ tätig zu werden. Der Senat orientiert sich vielmehr an einer anderen privilegierenden Vorschrift des SGB V, nach der Bewerbern bei der Nachbesetzung (im Rahmen der Variante 2) bevorzugt werden, die bereits als Angestellte in der Praxis des bisherigen Vertragsarztes tätig waren oder die Praxis mit diesem gemeinschaftlich betrieben haben. Dort wird eine Kooperation mit einer Dauer von zumindest 3 Jahren vorgegeben, um zu verhindern, dass die Regelungen zum Abbau von Überversorgung durch ein nur kurzzeitiges Anstellungsoder Jobsharing-Verhältnis umgangen werden.

\section{Grundsatz:}

\section{Mindestens 3 Jahre Anstellung}

Diesen Gedanken überträgt das BSG nun auf die Anstellungsgenehmigung der Variante 3. Die Genehmigung werde dem MVZ nicht erteilt, um ihm die Möglichkeit zu geben, die Stelle ohne Bindung an die Auswahlentscheidung eines Zulas- sungsgremiums zu besetzen, nachzubesetzen oder in eine Zulassung umzuwandeln, sondern weil der Vertragsarzt dort als Angestellter tätig werden möchte. Nach Ablauf von 3 Jahren der Tätigkeit dieses Arztes im MVZ kann davon ausgegangen werden, dass die gesetzlich vorgegebene Gestaltung auch tatsächlich gewollt und gelebt worden sei.

\section{Vorzeitige Beendigung:}

\section{Nachweis der Absicht erforderlich}

Endet die Tätigkeit des Arztes, der unter Umwandlung seiner Zulassung in eine Anstellung bei einem MVZ „tätig werden wollte“, vor Ablauf von 3 Jahren, hänge das Nachbesetzungsrecht des MVZ davon $a b$, ob nach den Umständen davon ausgegangen werden kann, dass der ursprünglich zugelassene Arzt zunächst tatsächlich zumindest 3 Jahre im MVZ tätig werden wollte, diese Absicht aber aufgrund von Umständen, die ihm zum Zeitpunkt des Verzichts auf die Zulassung noch nicht bekannt waren, nicht mehr realisieren konnte. Das könne etwa der Fall sein, wenn er erkrankt oder aus zwingenden Gründen seine Berufs- oder Lebensplanung ändern musste.

Gegen den Willen zur Fortsetzung der vertragsärztlichen Tätigkeit im MVZ für zumindest 3 Jahre spreche dagegen z.B., wenn der Arzt im Zuge des Verzichts auf die Zulassung und der Beantragung der Anstellungsgenehmigung durch das MVZ schon konkrete Pläne für das alsbaldige Beenden seiner Tätigkeit entwickelt habe, oder wenn das MVZ zu diesem Zeitpunkt schon Verhandlungen mit einem an der Nachbesetzung der betroffenen Arztstelle interessierten anderen Arzt geführt habe, die sich auf die unmittelbare Zukunft und nicht auf einen erst in 3 Jahren beginnenden Zeitraum beziehen. Je kürzer die Angestelltentätigkeit des Arztes gewesen sei, desto höhere Anforderungen seien an den Nachweis der Umstände zu stellen, die die Absicht zur Ausübung der Angestelltentätigkeit für eine Dauer von zumindest 3 Jahren dokumentieren.

\section{Gelingt der Nachweis nicht - Arztstelle weg}

Wenn Änderungen der Verhältnisse, die eine Änderung der ursprünglich bestehenden Absichten nachvollziehbar erscheinen lassen, nicht festzustellen seien, gehe dies zu Lasten des an der Nachbesetzung der Arztstelle interessierten MVZ. Dies hat dann zur Folge, dass der Zulas- 
sungsausschuss die beantragte (vorzeitige) Nachbesetzung ablehnen muss und die Arztstelle verfällt.

\section{Ausnahme bei schrittweiser Reduzierung im Rahmen eines Altersmodells}

Um auch die Interessen der Ärztinnen und Ärzte zu wahren, die zwar tatsächlich noch in einem MVZ tätig werden, altersbedingt aber ihren Tätigkeitsumfang allmählich vermindern wollten, könne sich die angestrebte Anstellung für eine Dauer von wenigstens 3 Jahren als zentraler Indikator für den erforderlichen Tätigkeitswillen nur auf die Tätigkeit als solche beziehen. Wenn also ein solcher Arzt zunächst ein Jahr in dem Umfang im MVZ tätig gewesen sei, in dem er zuvor als zugelassener Arzt an der Versorgung teilgenommen habe, seinen Beschäftigungsumfang in den beiden folgenden Jahren aber vermindere, etwa indem er jeweils seinen Beschäftigungsumfang schrittweise um den Anrechnungsfaktor $1 / 4$ reduziert, wirke sich dies nicht auf das Nachbesetzungsrecht des MVZ aus, sodass insoweit die allgemeinen Regelungen gelten würden (vgl. dazu auch das weitere Urteil des BSG vom 04.05.2016, Az: B 6 KA 28/15 R).

Ab wann gilt die 3-Jahre-Vorgabe? Die strikte Ausrichtung des Willens zum „Tätigwerden“ in einem MVZ nach Verzicht auf die Zulassung an der Dauer einer Angestelltentätigkeit von wenigstens 3 Jahren gilt aus Gründen des Vertrauensschutzes uneingeschränkt erst für Nachbesetzungen, die sich auf Arztstellen beziehen, denen Umwandlungsanträge (nicht Nachbesetzungsanträge!, Anm. der Verf.) von Ärzten aus der Zeit nach Verkündung dieses Urteils zu Grunde liegen.

\section{„Beweislastumkehr“ fürr Altfälle}

Für die Fälle, in denen bereits vor Urteilsverkündung Umwandlungsanträge gestellt wurden und eine Nachbesetzung vor Ablauf der 3 Jahre beantragt wird, gilt aber nicht etwa jede Konstellation als zulässig. Vielmehr sei unter Würdigung aller maßgeblichen Umstände von den Zulassungsgremien zu prüfen, ob hinreichende Anhaltspunkte dafür vorliegen, dass der früher zugelassene Arzt, der vor Ablauf von 3 Jahren seine Tätigkeit im MVZ wieder beendet hat, dort nicht zumindest eine gewisse Zeit tätig werden wollte. Lassen sich Zweifel an einer ent- sprechenden Absicht des Arztes nicht hinreichend verifizieren, gehe das zu Lasten der Zulassungsgremien, die dem MVZ die Nachbesetzung der betroffenen Arztstelle - ganz oder mit vermindertem Anrechnungsfaktor - dann nicht versagen dürften.

\section{Gefahr des Arztstellenverlustes auch bei Altfällen}

Das BSG zieht auch bei den Altfällen eine Art Untergrenze ein. Die Arztstelle kann nach Auffassung des BSG nur nachbesetzt werden, wenn der Arzt, der auf seine Zulassung verzichtet habe, überhaupt als Angestellter im MVZ tätig werden wollte. Die Nachbesetzung soll grundsätzlich auch nur in dem Umfang möglich sein, in dem der Arzt seine Tätigkeit im MVZ aufgenommen habe. War demnach ein Arzt überhaupt nicht tätig geworden, entfällt die Nachbesetzungsmöglichkeit. War er in geringerem Umfang als mit einer ganzen Stelle angestellt worden, ist eine Nachbesetzung in dem von vornherein nicht in Anspruch genommenen Umfang der Beschäftigung ausgeschlossen.

\section{Rechtsprechung gilt nicht für weitere Nachbesetzungen}

Ausdrücklich stellt der Senat klar, dass die neue Rechtsprechung grundsätzlich keine Auswirkungen auf die Genehmigung einer erneuten Nachbesetzung von (ursprünglich umgewandelten Vertragsarztsitzen durch Verzicht zugunsten einer Anstellung) Stellen hat, die schon einmal nachbesetzt worden waren. Hat der Zulassungsausschuss also die erstmalige Nachbesetzung einer vollen oder anteiligen Arztstelle, die ursprünglich aus der Umwandlung einer Zulassung beim MVZ entstanden ist, nach dem Ausscheiden des ursprünglich zugelassenen und später angestellten Arztes aus dem MVZ bestandskräftig genehmigt, könne dem Antrag auf erneute Nachbesetzung dieser Stelle (voll oder zu verschiedenen Anteilen) nicht entgegengehalten werden, die vorangegangene Nachbesetzung sei bereits zu Unrecht genehmigt worden. Auf eine bestandskräftig erteilte Anstellungsgenehmigung könne im Regelfall auch eine darauf folgende Nachbesetzung gestützt werden. Schutzwürdig sei damit auch die Position des MVZ, dem bestandskräftig die Nachbesetzung einer Arztstelle genehmigt worden sei, die ursprünglich aus einer umgewandelten Zulassung entstanden und bereits einmal nachbesetzt worden war. Die Stelle könne beim Ausscheiden dieses Arztes auch dann (erneut) nachbesetzt werden, wenn Zweifel daran bestünden, ob die erste Nachbesetzung hätte genehmigt werden dürfen, weil beim MVZ keine (volle oder anteilige) Arztstelle entstanden sei.

\section{Fazit}

Mit dem Urteil greift das BSG massiv in die bisherige Spruchpraxis der Zulassungsgremien ein. Über allem schwebt der Gedanke des Abbaus der Überversorgung und der Wunsch, (auch gesetzlich vorgesehene) Umgehungen so weit wie möglich einzudämmen. Die jüngste Spruchpraxis der Zulassungsgremien nach Verkündung des Urteils zu den „Altfällen“ lässt hoffen, dass aufgrund des zeitlich überraschenden Urteils nicht rigide der Überversorgungsabbau betrieben wird. Es empfiehlt sich der enge Austausch mit den Zulassungsgremien vor Stellung eines Nachbesetzungsantrags in kritischen Fällen. Erhebliche Probleme wirft das Urteil für ältere Vertragsärzte auf, die im Vertrauen auf die bisherige Spruchpraxis von einer zulässigen kurzen Anstellungszeit ausgegangen sind und denen eine dreijährige (auch schrittweise reduzierte) Tätigkeit schlichtweg nicht zugemutet werden kann. Bereits jetzt zeichnet sich $a b$, dass in diesen Fällen wieder der klassische Weg der Ausschreibung beschritten wird (Variante2). Dringend abzuraten ist von (fingierten) Anstellungsverträgen mit Mindestlaufzeit von 3 Jahren bei besserem Wissen, dass diese 3 Jahre nicht eingehalten werden sollen. Werden Anhaltspunkte dafür sichtbar, riskiert das MVZ u.a., die Arztstelle zu verlieren. Besondere Anforderungen erwachsen aus der Rechtsprechung an die Vertragsgestaltungen.

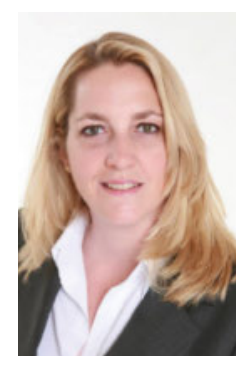

Korrespondenz Dr. iur. Isabel Häser Rechtsanwältin Fachanwältin für Medizinrecht Haimhauser Str. 1 80802 München E-Mail: haeser@kanzleihaeser.de 